\title{
Immunological assessment of plant-derived avian flu H5/HA1 variants.
}

S Spitsin

Thomas Jefferson University

V Andrianov

Thomas Jefferson University

N Pogrebnyak

Thomas Jefferson University

Y Smirnov

Thomas Jefferson University

N Borisjuk

Thomas Jefferson University Follow this and additional works at: https://jdc.jefferson.edu/cbfp

Part of the Amino Acids, Peptides, and Proteins Commons

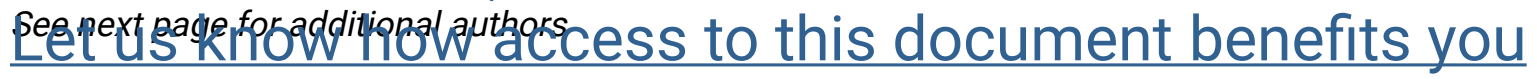

\section{Recommended Citation}

Spitsin, S; Andrianov, V; Pogrebnyak, N; Smirnov, Y; Borisjuk, N; Portocarrero, C; Veguilla, V; Koprowski, H; and Golovkin, M, "Immunological assessment of plant-derived avian flu H5/HA1 variants." (2009). Department of Cancer Biology Faculty Papers. Paper 18.

https://jdc.jefferson.edu/cbfp/18

This Article is brought to you for free and open access by the Jefferson Digital Commons. The Jefferson Digital Commons is a service of Thomas Jefferson University's Center for Teaching and Learning (CTL). The Commons is a showcase for Jefferson books and journals, peer-reviewed scholarly publications, unique historical collections from the University archives, and teaching tools. The Jefferson Digital Commons allows researchers and interested readers anywhere in the world to learn about and keep up to date with Jefferson scholarship. This article has been accepted for inclusion in Department of Cancer Biology Faculty Papers by an authorized administrator of the Jefferson Digital Commons. For more information, please contact: JeffersonDigitalCommons@jefferson.edu. 
Authors

S Spitsin, V Andrianov, N Pogrebnyak, Y Smirnov, N Borisjuk, C Portocarrero, V Veguilla, H Koprowski, and M Golovkin 
Short Communication

As submitted to: Vaccine and later published as:

"Immunological assessment of plant-derived avian flu H5/HA1 variants." In Vaccine. Volume 27, Issue 9, 25 February 2009, Pages

1289-1292

DOI: 10.1016/j.vaccine.2008.12.050

\title{
Immunological Assessment of Plant-derived Avian Flu H5/HA1 Variants
}

\author{
S. Spitsin ${ }^{1}$, V. Andrianov ${ }^{1}$, N. Pogrebnyak ${ }^{1}$, Y.Smirnov $^{1}$, N. Borisjuk ${ }^{1}$, C. \\ Portocarrero $^{1}$, V. Veguilla ${ }^{2}$, H. Koprowski ${ }^{1}$ and M. Golovkin ${ }^{1} *$ \\ ${ }^{1}$ Biotechnology Foundation Laboratories, Thomas Jefferson University, Philadelphia, PA \\ 19107-6799 \\ ${ }^{2}$ Centers for Disease Control and Prevention, Atlanta, GA 30333
}

*to whom correspondence should be addressed:

Ph. 215-503-1620; Fax. 215-503-6795; E-mail: maxim.golovkin@jefferson.edu

Number of pages: $\quad \mathbf{1 0}$

Number of figures: $\quad 2$

Number of words in the abstract: $\quad \mathbf{1 0 7}$

Number of characters in the paper: $\quad 10343$ 
Abbreviations: hemagglutinin, $\boldsymbol{H A}$; plant-derived HAl antigen, $\mathbf{p H A}$; $p H A 1$ fused with mouse or human heavy chain IgG Fc fragment, pHA1-mFc or pHA1-hFc;

Key words: $\quad$ Plant biotechnology/ avian flu H5N1/recombinant subunit vaccine/ 


\begin{abstract}
Polypeptide variants of the HA1 antigenic domain of the H5N1 avian influenza virus hemagglutinin (HA) molecule were produced in plants using transient and stable expression systems and fused with His/c-myc tags or with mouse or human Fc antibody fragments. The resulting peptides were purified and used for intramuscular immunization of mice. While the recombinant HA1 variants induced a significant serum humoral immune response in the mice, none of the HA1 preparations induced virus-neutralizing antibodies. Fusion with the Fc fragment improved overall yield of the constructs and allowed purification requiring only a single step, but led to no detectable fusion-related enhancement of immunogenicity or quality of immune response.
\end{abstract}




\section{INTRODUCTION}

The development of cost-effective avian flu vaccines for both humans and domestic poultry is an urgent priority to prevent pandemic flu outbreaks [1]. Recombinant subunit vaccines that incorporate antigenic viral membrane glycoproteins, such as hemagglutinin (HA), are particularly attractive candidates, since these molecules can induce virus-neutralizing antibodies [2]. The HA1 antigenic domain of HA has been shown to induce an immune response equal to that of the full-size protein [3, 4]. Here, we describe the successful production of recombinant HA1 antigenic variants using transient and stable plant expression systems, a promising alternative to microbial and/or animal pharmaceutical protein production systems [5].

\section{MATERIALS AND METHODS}

\subsection{Expression of HA1 variants in plants}

The cDNA template of the HA from A/Viet Nam/1203/2004 (H5N1), obtained from CDC (Atlanta, GA), was used for PCR-based cloning of the following HA constructs (Fig. 1A): the full-size mature HA without viral leader peptide (aa 1-549); $37 \mathrm{kDa}$ HA1 polypeptide containing major HA antigenic domains (aa 1-330); and two shorter versions, $34 \mathrm{kDa}$-lacking C-terminal fragment containing cleavage site and sequences with potential poor solubility (aa 1-277) and $27 \mathrm{kDa}$ fragment lacking N-terminal part (aa 68-277). A set of appropriate PCR primers carrying restriction site adapters NcoI (forward) and NotI (reverse) were used to clone each variant of the HA gene in-frame with C-terminal His- and c-myc tags of the Impact Vectors system used for stable plant transformation (Plant Research International, Wageningen, Netherlands). For some of the variants, compatible restriction sites of transient MagnIcon pro-vector pICH11599 (IconGenetics, Halle, Germany) were used to expedite expression [6]. To generate HA1-Fc fusions (HAl-Fc), cDNA clones encoding the heavy chain of human anti-rabies monoclonal antibody SO57 [7] and the heavy chain of murine antibody CO17-1A [8] were used as PCR templates. HA1 and Fc fragments were joined by a flexible bridge of seven amino acids \{GGGSGNS\}. Both human $(H A l-h F c)$ and mouse $(H A l-m F c)$ fusions were arranged using the Impact Vector system (tagged vectors \# 1.1 and 1.3). The resulting cassettes carrying two 
affinity-purification tags (c-myc and 6xHis) at the C-terminus were subcloned into the Impact binary vector AscI-PacI restriction sites and used for Agrobacterium-mediated stable transformation of tobacco plants as described [9]. The plant signal peptide for apoplast secretion and the HDEL C-terminal ER retention signal were used for transient (Nicotiana benthamiana) and stable (Nicotiana tabacum) expression, respectively.

\subsection{Isolation and purification of recombinant proteins}

$H A$-based polypeptide variants were isolated from plant tissues and purified by a 2 -step procedure using His- and c-myc tags exactly as described [6]. HA-Fc fusion proteins were purified in a single-step protocol using protein $\mathrm{A}$ beads as described [7]. In vitro characterization and quantification of protein expression were done by ELISA and Western blot analysis as described [6, 7]. Full-size baculovirus-expressed H5 protein from BEI Resources (Manassas, VA) was used as positive control.

\subsection{Immunological assessment of HA1 variants in mice}

Six- to 8-week-old female BALB/c mice (5 per group) were immunized intramuscularly with recombinant peptides 2 times (two weeks interval) at a dose of $10 \mu \mathrm{g}$ per immunization using alum-CpG adjuvant essentially as described [6]. Ten days after the last immunization, mice were sacrificed and sera from individual mice were analyzed by ELISA and Western blotting as described [6], followed by virus micro-neutralization and hemagglutination inhibition assays [10]. Results are expressed as the mean $\pm \mathrm{SD}$ for groups of five mice. All animal experiments were conducted in accordance with the institutional guidelines for animal welfare.

\section{RESULTS AND DISCUSSION}

Initial attempts to express the entire H5 protein $(\sim 65 \mathrm{kDa})$ or its $H A 1$ domain $(\sim 37 \mathrm{kDa})$ in plants yielded minor quantities of the recombinant material by stable and/or transient transformation procedures (data not shown). Further testing revealed that the major part of HAl containing the antigenic region can be efficiently expressed either as a truncated 34or $27-\mathrm{kDa}$ protein by transient system (Fig. 1A, B) or as a $37-\mathrm{kDa}$ polypeptide fused at the C-terminus with a 26-kDa human or mouse $\mathrm{Fc}$ fragment (Fig. 1C) by stable transformation. 
All recombinant plant-produced proteins pHA1 and pHA1-Fc were present in the soluble protein fraction and showed the expected molecular weight of the resulting products. Magnicon-based expression of $34 \mathrm{kDa} H A 1$ yielded up to $1 \mathrm{mg} / \mathrm{kg}$ of fresh tissue. Stably transformed tobacco plants ( $>15$ independent transgenic lines) confirmed high-level expression of recombinant Fc-fusion proteins, i.e., up to $4 \mathrm{mg} / \mathrm{kg}$ of fresh tissue, as determined by Western blot analysis using c-myc- (Fig. 1C) or HA1-specific antibodies (not shown). Intense secondary bands resolved from the Fc fusion constructs suggested a propensity for cleavage/degradation, especially in older leaf material (Fig. 1C, lanes 1b-3b).

The pHA1 fragments produced by the MagnICON system were purified using a 2-step $\mathrm{Ni}^{++}$and c-myc chromatography procedure, while pHA1-Fc constructs were purified using single-step protein A chromatography (Fig. 1D, E). The purification procedure increased degradation of both human and mouse Fc-fused polypeptides. In Western blots the c-myc-detectable degradation product of approximately $40 \mathrm{kDa}$ is the result of the HA1/HA2 cleavage site left 97 aa upstream of the Fc. Addition of protease inhibitor cocktail (Sigma) during purification improved stability only marginally. Nevertheless, purification of HA1-Fc constructs by simple single-step protein-A chromatography yielded around $2 \mathrm{mg}$ of $\mathrm{HA} 1-\mathrm{Fc} / \mathrm{kg}$ of fresh plant tissue (50\% yield).

\subsection{Immunogenicity of $\mathrm{HA1}$ protein in mice}

The purified peptides from the $34 \mathrm{kDa} \mathrm{HA} 1$ and $\mathrm{HA} 1-\mathrm{Fc}, \mathrm{HA} 1-\mathrm{mFc}$ fusions were used to immunize mice at similar doses ( $10 \mu \mathrm{g}$ per immunization). Dosage for HA1-Fc constructs was calculated based on Western blot band intensity of commercial proteins. ELISA analysis revealed a strong H5-specific serum IgG response to all three HA1 constructs (Fig. 2A), and Western blot analysis confirmed the specificity of the serum immune response against baculovirus-expressed full-size H5 and plant-derived HA1 (Fig. 2B). High titers of mouse anti-human IgG were also detected in sera of mice immunized with pHA1-hFc (Fig. 2C).

Several recent reports demonstrated that HA and HA1 fragment containing the majority of antigenic determinants are responsible for generation of virus-neutralizing antibodies [4, 11-15]. A transient expression system was successfully used to produce in 
plants HA from A/Wyoming/03/03 (H3N2) virus that generated neutralizing antibodies in mice [11]. However, in our plant-based system, despite the high serum titers of H5-specific IgG in mice immunized with all three HA1 variants, none of the sera demonstrated neutralizing activities in a virus micro-neutralization assay (all titers less than 20) or hemagglutination inhibition assay (all titers less than 10). Moreover, although fusion of antigen with the $\operatorname{IgG~Fc}$ domain may increase expression levels, improve stability and, in some cases, increase immunogenicity of antigens [16], we did not detect improvement of immunogenicity and with mouse Fc fusion observed a slight decrease of HA1 immunogenicity. The ease of purification procedure as a function of this fusion was confirmed. At present time we can not pinpoint the reason for the lack of neutralizing activity in our case. The other research differs either in selection of virus strain(s) and/or the area of HA polypeptides expressed. There are also differences to the delivery systems, such as subunit vaccines, DNA vaccines and viral vectors; choice of adjuvants, etc. The question also remains to whether lack of neutralizing antibodies is due to the dosage, peptide folding, purity etc. $[12,17,18]$. All these may have an impact on the ability of HA to generate a protective antibody response. Lower immunogenicity of H5 may be resolved by using better adjuvant(s) and/or different methodology of administration [19]. Further research is needed to optimize production of H5-derived antigens in plants.

Conclusions: both the Magnicon transient system and Fc-fusion served to improve yield of viral glycoproteins otherwise difficult to express in plants. Fusion of HA1 fragments with the Fc region of human or mouse IgG heavy chains allowed simple protein A-based purification of the final product but did not have a significant impact on immunogenicity or quality of the immune response against H5.

\section{ACKNOWLEDGEMENTS}

We thank A. Jakubowski and K. Markley for technical help. This work was supported by a grant from Commonwealth of Pennsylvania Department of Health to Biotechnology Foundation Laboratories (to H.K.) The findings and conclusions in this report are those of the authors and do not necessarily represent the views of the Centers for Disease Control and Prevention. 


\section{REFERENCES}

1. Webster RG, Govorkova EA. H5N1 influenza - continuing evolution and spread. N Engl J Med 2006; 355(21):2174-2177.

2. Bardiya N, Bae JH. Influenza vaccines: recent advances in production technologies. Appl Microbiol Biotechnol 2005; 67:299-305.

3. Caton AJ, Brownlee GG, Yewdell JW, and Gerhard W. The antigenic structure of the influenza virus A/PR/8/34 hemagglutinin (H1 subtype). Cell 1982; 31:417-427.

4. Tonegawa K, Nobusawa E, Nakajima K, Kato T, Kutsuna T, Kuroda K, Shibata T, Harada Y, Nakamura A and Itoh M. Analysis of epitope recognition of antibodies induced by DNA immunization against hemagglutinin protein of influenza A virus. Vaccine 2003; 21:3118-3125.

5. Rybicki EP. Plant-produced vaccines: promise and reality. Drug Discovery Today 2008; available online: doi:10.1016/j.drudis.2008.10.002.

6. Golovkin M, Spitsin S, Andrianov V, Smirnov Y, Xiao Y, Pogrebnyak N, Markley K, Brodzik R, Gleba Y, Isaacs SN, and Koprowski H. Smallpox subunit vaccine produced in planta confers protection in mice. Proc Natl Acad Sci USA 2007:104; 6864-6869.

7. Ko K, Tekoah Y, Rudd PM, Harvey DJ, Dwek RA, Spitsin S, Hanlon CA, Rupprecht C, Dietzschold B, Golovkin M, Koprowski H. Function and glycosylation of plant-derived antiviral monoclonal antibody. Proc Natl Acad Sci U S A. 2003; 100(13):8013-8018.

8. Ko K, Steplewski Z, Glogowska M, Koprowski H. Inhibition of tumor growth by plant-derived mAb. Proc Natl Acad Sci U S A. 2005; 102(19):7026-7030.

9. Pogrebnyak N, Golovkin M, Andrianov V, Spitsin S, Smirnov Y, Egolf R, Koprowski H. Severe acute respiratory syndrome (SARS) S protein production in 
plants:development of recombinant vaccine. Proc Natl Acad Sci U S A. 2005; 102(25):9062-9067.

10. Rowe T, Abernathy RA, Hu-Primmer J, Thompson WW, Lu X, Lim W, Fukuda K, Cox NJ and Katz JM. Detection of antibody to avian influenza A (H5N1) virus in human serum by using a combination of serologic assays. J Clin Microbiol 1999;37:937-943.

11. Shoji Y, Chichester JA, Bi H, Musiychuk K, de la Rosa P, Goldschmidt L, Horsey A, Ugulava N, Palmer GA, Mett V and Yusibov V. Plant-expressed HA as a seasonal influenza vaccine candidate. Vaccine 2008; 26;2930-2934.

12. Wei CJ, Xu L, Kong WP, Shi W, Canis K, Stevens J, Yang ZY, Dell A, Haslam SM, Wilson IA, Nabel GJ. Comparative efficacy of neutralizing antibodies elicited by recombinant hemagglutinin proteins from avian H5N1 influenza virus. J Virol 2008; 82(13):6200-6208.

13. Okamoto S, Yoshii H, Akagi T, Akashi M, Ishikawa T, Okuno Y, Takahashi M, Yamanishi K, Mori Y. Influenza hemagglutinin vaccine with poly(gamma-glutamic acid) nanoparticles enhances the protection against influenza virus infection through both humoral and cell-mediated immunity. Vaccine 2007; 25(49):8270-8278.

14. Garg S, Hoelscher M, Belser JA, Wang C, Jayashankar L, Guo Z, Durland RH, Katz JM, Sambhara S. Needle-free skin patch delivery of a vaccine for a potentially pandemic influenza virus provides protection against lethal challenge in mice. Clinical \& Vaccine Immunology 2007; 14(7):926-928.

15. Kreijtz JH, Suezer Y, van Amerongen G, de Mutsert G, Schnierle BS, Wood JM, Kuiken T, Fouchier RA, Lower J, Osterhaus AD, Sutter G, Rimmelzwaan GF. Recombinant modified vaccinia virus Ankara-based vaccine induces protective immunity 
in mice against infection with influenza virus H5N1. J Infect Dis 2007; 195(11):1598-1606.

16. Obregon P, Chargelegue D, Drake PM, Prada A, Nuttall J, Frigerio L and Ma JK. HIV-1 p24-immunoglobulin fusion molecule: a new strategy for plant-based protein production. Plant Biotechnol J 2006; 4:195-207.

17. Treanor JJ, Campbell JD, Zangwill KM, Rowe T, Wolff M. Safety and Immunogenicity of an Inactivated Subvirion Influenza A (H5N1) Vaccine. Engl J Med 2006; 354(13):1343-1351.

18. Portocarrero C, Markley K, Koprowski H, Spitsin S, Golovkin M. Immunogenic properties of plant-derived recombinant smallpox vaccine candidate pB5. Vaccine 2008; 26(43):5535-5540.

19. Stephenson I, Bugarini R, Nicholson KG, Podda A, Wood JM, Zambon MC, Katz JM. Cross-reactivity to highly pathogenic avian influenza H5N1 viruses after vaccination with nonadjuvanted and MF59-adjuvanted influenza A/Duck/Singapore/97 (H5N3) vaccine: a potential priming strategy. J Infect Dis 2005;191(8):1210-1215

\section{FIGURE LEGENDS}

Figure 1. Expression and purification of HA1, HA1-hFc and HA1-mFc polypeptides.

(A) HA1 glycoprotein cloning strategy. (I) Schematic diagram of the full-length H5 protein comprised of a HA1, HA2 domains and cleavage site (shaded square). Putative glycosylation sites are indicated with asterisks; 37-kDa HA1 peptide (aa 1-330); 34-kDa peptide (aa 1-277); and 27-kDa peptide (aa 68-277). (II) 37-kDa HA1 peptide (aa 1-330) linked to the heavy chain Fc-fragment of either mouse (IgG-mFc) or human (IgG-hFc) immunoglobulin. (B) Western blot analysis of plant-derived (transient transformation) 34- 
and 27-kDa HA1 peptides. Equal amounts of total soluble protein (TSP) extracted from two independent transfection samples were loaded onto the SDS-polyacrylamide gel and analyzed by Western blotting using c-Myc Mab. (C) Western blot analysis of plant-derived HA1-hFc after stable transformation, detected in TSP extracts of three independent transgenic lines by c-Myc Mab. Bands of the expected molecular weight are seen in young (a) leaves (arrowhead) and some degradation products are seen in old (b) leaves (double arrowheads). (D) Western blot analysis of two representative plant-derived purified samples HA1-hFc (pHA1-hFc) and HA1-mFc (pHA1-mFc) using c-myc antibodies and HA1-specific antibodies (data not shown). Full-size H5 (+) containing c-myc tag was used as positive control. Products of expected size are marked with arrowhead. Sub-products (double arrowheads) were detected in both HA1-Fc and commercial H5. (E) Coomassie-stained gel showing the resolved purified batches of $\mathrm{pHA} 1-\mathrm{hFc}$ as compared to commercial IgG (heavy and light chain) titration.

\section{Figure 2. Serum antibody response against pHA1-based polypeptides in mice.}

(A) H5-specific IgG titers after second immunization of mice with $34 \mathrm{kDa} \mathrm{pHA} 1$, pHA1-mFc and pHA1-hFc as determined in ELISA against the full-size H5 from BEI Resources (Manassas, VA). (B) Western blot analysis of pooled mouse sera (pHA1-mFc, pHA1-hFc or $34 \mathrm{kDa}$ pHA1, see panel A) using the commercial full-size H5 preparation and the plant-derived HA1-hFc. Right panel shows detection of pHA1-hFc with c-Myc Mab. (C) ELISA titers, determined using mouse anti-human $\mathrm{IgG}$, obtained after second immunization with pHA1-hFc. 